\title{
Role of Lamellar Hole-Associated Epiretinal Proliferation in Lamellar Macular Holes
}

\section{ROBERTO DELL'OMO, GIANNI VIRGILI, STANISLAO RIZZO, SERENA DE TURRIS, GIOVANNI COCLITE, DARIO GIORGIO, ERMANNO DELL'OMO, AND CIRO COSTAGLIOLA}

- PURPOSE: To compare the morphologic and functional characteristics and response to surgery of lamellar macular holes (LMHs) with and without lamellar hole-associated epiretinal proliferation (LHEP) and standard epiretinal membrane (ERM).

- DESIGN: Retrospective observational case series.

- METHODS: SETTING: Vitreoretinal clinical practice. Study Population: Eigthy-four eyes of 84 patients. The included eyes must present an irregular foveal contour and schitic or cavitated lamellar separation of neurosensory retina on spectral-domain optical coherence tomography (SDOCT) and an area of increased autofluorescence on blue fundus autofluorescence (B-FAF). Twenty-six eyes underwent pars plana vitrectomy (PPV). MAIN OUtcome MEAsuRes: Logarithm of minimum angle of resolution (logMAR) best-corrected visual acuity (BCVA) and evolution of morphologic characteristics.

- RESULTS: Standard ERM alone, LHEP alone, and concomitant ERM and LHEP were found in 51.2\%, $13.1 \%$, and $35.7 \%$ of the cases, respectively. A substantial stability of functional and morphologic parameters throughout the follow-up period was observed in the eyes that did not undergo surgery indipendently from the associated epiretinal material. The most significant change, observed in the preoperative period, in the eyes that underwent surgery, was the thinning of the central foveal thickness (CFT, $P<.001)$. In the operated eyes, logMAR BCVA continuosly improved during the postoperative period $(P=.006)$, CFT was found increased, and diameters of the hole were found reduced since the first month after operation $(P<.001)$.

- CONCLUSIONS: In eyes with LMHs, presence of LHEP without any trace of standard ERM is rare. The presence of LHEP does not seem to influence the natural course of the disease and the response to surgery. (Am J Ophthalmol 2017;175:16-29. (C) 2016 Elsevier Inc. All rights reserved.)

Accepted for publication Nov 10, 2016.

From the Department of Medicine and Health Sciences "V. Tiberio", University of Molise, Campobasso, Italy (R.d'O., S.D.T., G.C., C.C.); Department of Translational Surgery and Medicine, University of Florence, Florence, Italy (G.V., S.R., D.G.); and Casa di Cura "Villa Maria”, Campobasso, Italy (R.d'O., E.d'O., C.C.).

Inquiries to Roberto dell'Omo, Department of Medicine and Health Sciences "V. Tiberio", University of Molise, Via Francesco De Sanctis 1, 86100 Campobasso, Italy; e-mail: roberto.dellomo@unimol.it

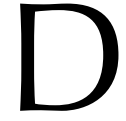

ESPITE CONTINUOUS IMPROVEMENTS IN IMAGING techniques, a clear distinction between subtypes of lamellar macular holes (LMHs) and the epiretinal materials associated with these conditions remains difficult. Based on spectral-domain optical coherence tomography (SDOCT), LMHs are characterized by an irregular foveal contour, intraretinal splitting or cavitation, and intact or disrupted outer retinal layers. ${ }^{1-4}$ The associated epiretinal material can be thin and highly reflective (the so-called standard epiretinal membrane [ERM]) or of medium reflectivity with focal variations in thickness (the so-called lamellar hole-associated epiretinal proliferation [LHEP]).

Histopathologic analysis has shown that myofibroblasts dominate in highly reflective membranes, whereas membranes of medium reflectivity consist primarily of fibroblasts and hyalocytes. ${ }^{10,11}$ As a consequence, it has been hypothesized that membranes of medium reflectivity should possess weaker contractive properties than standard ERMs.

Surrogates that estimate tractional changes induced by an epiretinal material by using OCT include wrinkling of the retinal surface, alteration of the foveal contour, retinal thickening, and disruption of the outer retinal bands. All of these features are difficult to quantify, and their detection is strictly related to the methodology used ${ }^{2}$ (density, orientation, and the number of B-scans recorded). In addition, none of these features is pathognomonically associated with one specific type of epiretinal material found in conjunction with LMHs.

Independent of the characteristics of the associated epiretinal materials and their alleged contractile properties, LMHs usually remain stable over time, with very few LMHs evolving into full-thickness macular holes. ${ }^{6,7,12}$

Blue fundus autofluorescence (B-FAF) imaging is a modality that relies on the fluorescence generated by the bisretinoids of lipofuscin in retinal pigment epithelial (RPE) cells and is influenced by absorbent or autofluorescent materials anterior to the RPE monolayer. ${ }^{13,14}$

When imaged with B-FAF imaging, LMHs are characterized by an increased autofluorescent signal, the intensity of which does not correlate with the thickness of the residual outer retinal tissue. ${ }^{15}$ The size of the areas of increased B-FAF related to different subtypes of LMHs, their evolution over time, and their relationships with different types of epiretinal material and other characteristics recordable on OCT have not been investigated in detail. 
In addition, the potential influence of different types of $\mathrm{LMHs}$ and their related epiretinal materials on outcomes after surgery have not been studied using OCT and B-FAF simultaneously.

The purpose of this study was to investigate the natural evolution of morphologic characteristics and outcomes after surgery in eyes with $\mathrm{LMH}$ s using both OCT and B-FAF imaging.

\section{METHODS}

WE CONDUCTED A RETROSPECTIVE CASE SERIES OF CONSECutive patients affected by LMHs seen at the University of Molise, Campobasso from January 1, 2008 to December 31, 2015. All subjects were treated in accordance with the Declaration of Helsinki. This study was approved by the Institutional Review Board of the University of Molise. Informed consent was obtained from all participants.

The SDOCT criteria used to diagnose LMHs were as follows: (1) an irregular foveal contour or a defect in the inner fovea; (2) lamellar separation of the neurosensory retina ("cavitated" or "schitic" in appearance) 9 in at least 1 horizontal or vertical scan; and (3) the absence of a fullthickness foveal defect.

The presence of a standard ERM was defined as a thin and highly reflective line, whereas LHEP was defined as a material of homogenous medium reflectivity measuring at least $20 \mu \mathrm{m}$ and located on the epiretinal surface. ${ }^{5}$

To be included in the study, the eyes must also present an area of increased autofluorescent signal that colocalized with the inner defect observed in the fovea and was not attributable to an intraretinal cyst based on OCT images.

The exclusion criteria were history of retinal detachment, advanced age-related macular degeneration, diabetic retinopathy, central retinal vein occlusion, uveitis, trauma, previous pars plana vitrectomy, and a refractive error of more than -8 diopters of spherical equivalent.

Patient characteristics, including age, sex, lens status, and refractive error, were recorded at the time of the first examination. The logarithm of the minimum angle of resolution ( $\log$ MAR) best-corrected visual acuity (BCVA) was tested at each visit using the Early Treatment Diabetic Retinopathy Study (ETDRS) chart at 4 meters, along with cataract progression in phakic eyes.

All images were collected using the Heidelberg Spectralis system (Heidelberg Engineering, Heidelberg, Germany), which combines an SDOCT with a confocal scanning laser ophthalmoscope.

The "Follow-up" function that identifies previous scan locations and automatically guides the OCT instrument to scan the same area for subsequent visits was used from the time of the first SDOCT scan to the time of the most recent encounter. The OCT recording protocol consisted of a sequence of 97 horizontal sections that covered an area of 30 or 20 degrees horizontally $\times 20$ degrees vertically and was recorded in the high-resolution mode (1536 and 1024 A-scans, respectively), with a distance of approximately $60 \mu \mathrm{m}$ between individual sections and at least 1 vertical scan passing through the fovea. Infrared, red-free and B-FAF (excitation wavelength at $488 \mathrm{~nm}$ and barrier filter at $500 \mathrm{~nm}$ ) images (35 degrees) were also obtained at each follow-up examination.

Multiple SDOCT morphologic characteristics were analyzed, including the relation of the vitreous cortex with the surface of the macula (attached/detached), the presence of retinal wrinkling, the presence of disruption of the external limiting membrane (ELM), ellipsoid and interdigitation zones (EZ and IZ), and central foveal thickness (CFT). The horizontal and vertical diameters of the holes were measured as the distance between the central borders of the outer plexiform layer, as shown in Figure 1. We decided to take measurements at this location because splitting or cavitation of the retina in eyes with LMHs typically occurs between the outer plexiform and outer nuclear layers. Furthermore, since B-FAF is attenuated by the presence of luteal pigment, which has a higher concentration along the outer plexiform layer at the fovea, we thought that examining hole diameters at this level with OCT could be of interest when compared with diameter measurements performed with B-FAF.

B-FAF was used to measure the horizontal and vertical diameters of the area of increased autofluorescence related to the lamellar hole (Figure 2) and to test the presence of retinal vessel printings (RVPs) (ie, lines of increased autofluorescence parallel to adjacent retinal vessels), which may be interpreted as a sign of tangential retinal traction in eyes with epiretinal membranes (Figure 3). ${ }^{16,17}$

Measurements of diameters were obtained manually with the Spectralis built-in manual caliper function on high magnification after adjusting the scale to $1: 1 \mathrm{~mm}$.

Anatomic progression was defined as widening of the hole by more than $50 \mu \mathrm{m}$, deepening of the hole by more than $20 \mu \mathrm{m}$, and/or the development of a full-thickness macular hole. Anatomic regression was defined as a reduction of the size or depth of the lesions.

Therefore, qualitative and quantitative analyses of the morphologic changes of the LMHs based on SDOCT and B-FAF findings were performed during a retrospective follow-up.

- SURGICAL PROCEDURE: Patients were recommended to surgery according to the following indication criteria: (1) a BCVA less than logMAR 0.3, (2) a BCVA that decreased 2 logMAR lines or more during the follow-up period, and (3) a subjective increase in metamorphopsia.

Twenty-five gauge pars plana vitrectomy was performed in all cases using a Constellation vitrectomy system (Alcon Labs, Fort Worth, Texas, USA). If necessary, a posterior vitreous detachment was induced via suction with the vitrectomy probe around the optic nerve head and then 

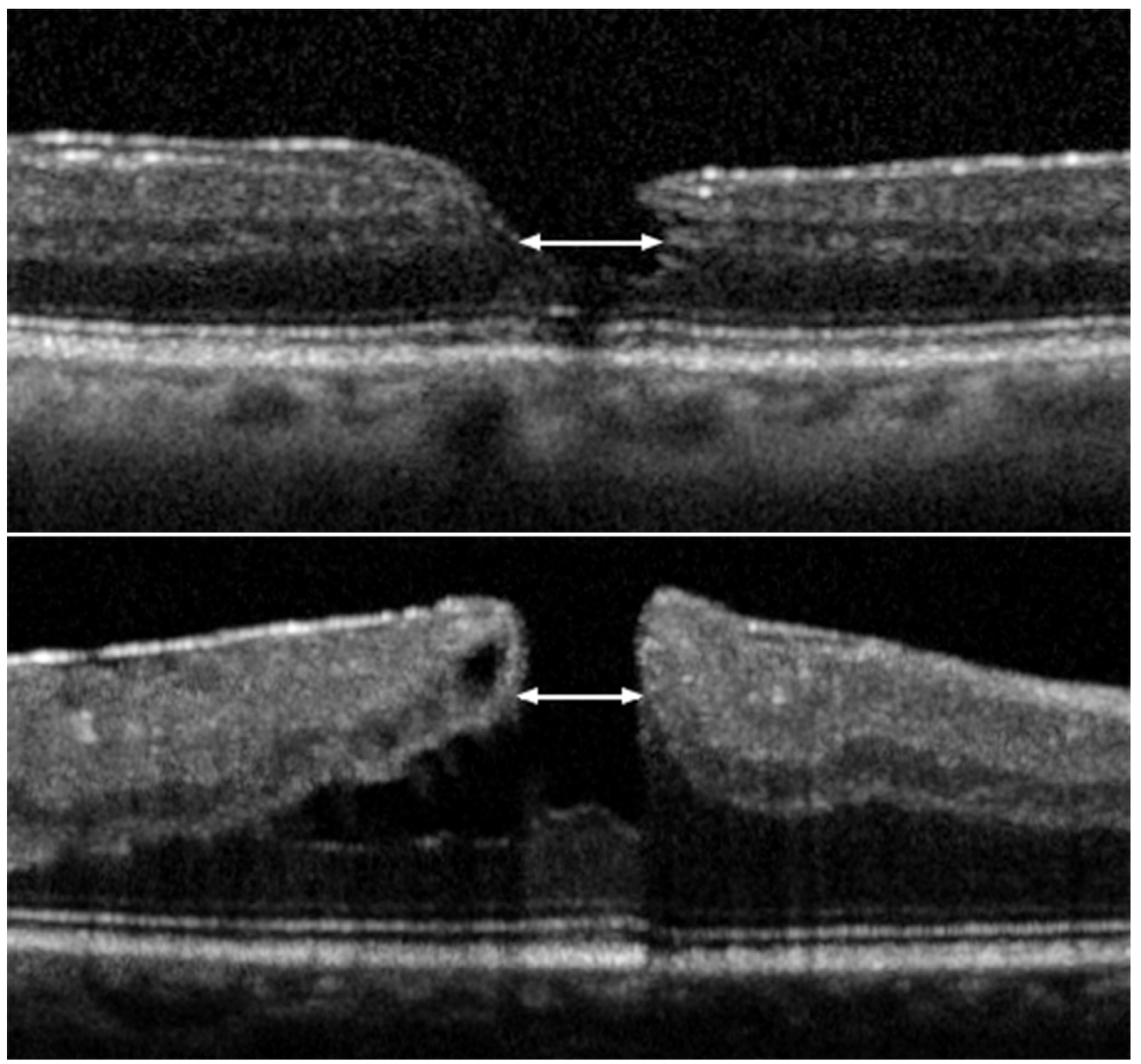

FIGURE 1. Optical coherence tomography measurements of diameters of lamellar macular holes (LMHs). The diameters are measured as the distance between the central borders of the outer plexiform layer. (Top) LMH with lamellar macular hole-associated epiretinal proliferation. (Bottom) LMH with standard epiretinal membrane.

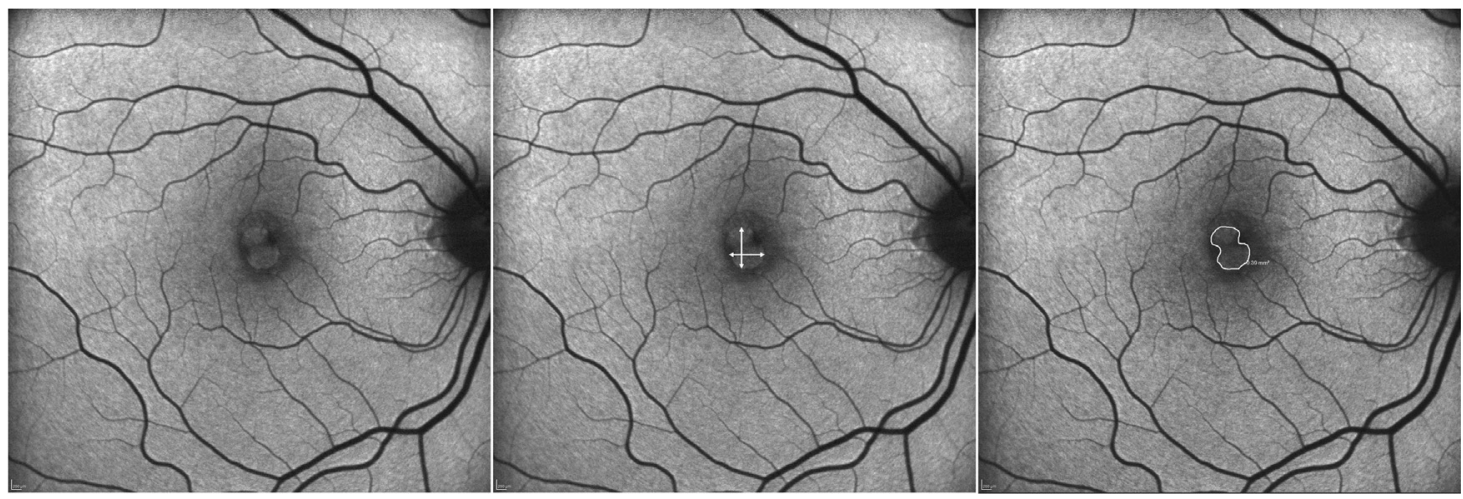

FIGURE 2. Blue-fundus autofluorescence-based measurements of lamellar macular holes. (Left) Area of hyperautofluorescence in the central macula, related to the lamellar hole. (Middle) Horizontal and vertical diameters of the hyperautofluorescent area. (Right) Measurement of the hyperautofluorecent area using the built-in software of the Spectralis.

extended at least up to the equator. A central and peripheral vitrectomy with shaving of the vitreous base were then carried out in all cases. After staining with Brilliant Blue
(Brilliant Blue G; DORC, Zuidland, The Netherlands), the epiretinal materials and internal limiting membranes (ILMs) were peeled from the macular area as far as the 


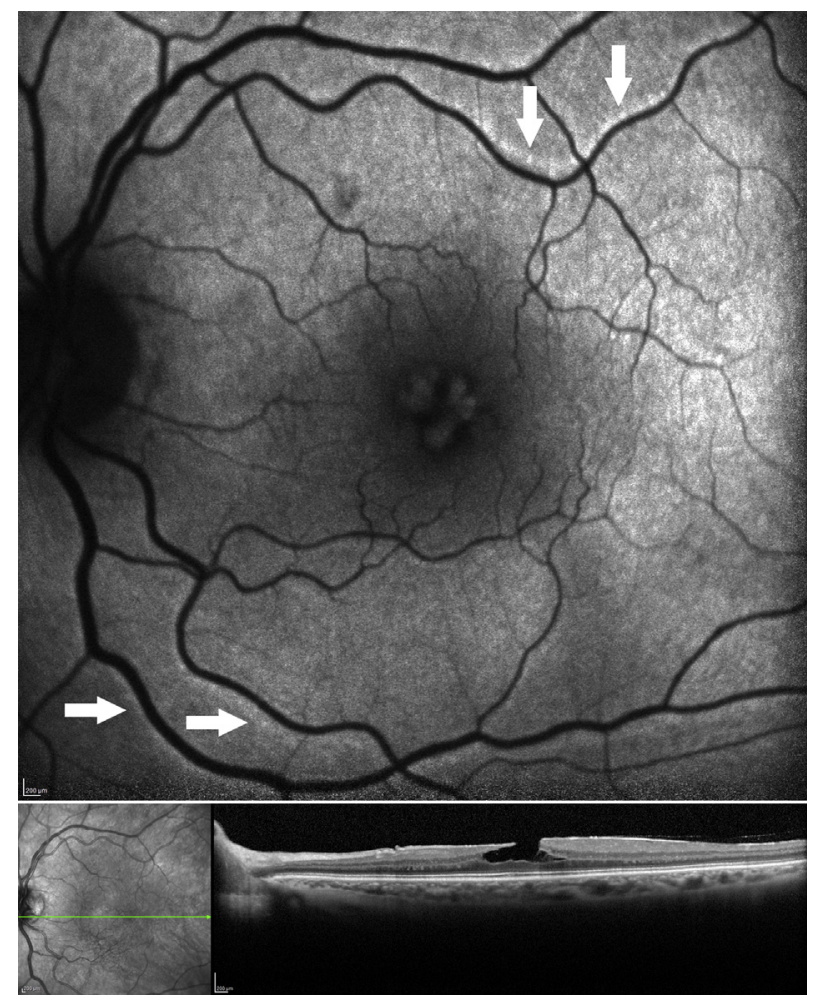

FIGURE 3. Retinal vessel printings (RVPs) seen in association with lamellar macular hole (LMH). (Top) On blue-fundus autofluorecence imaging, RVPs (arrows) appear as lines of increased autofluorescence parallel to the adjacent retinal vessels and are interpreted as a sign of tangential retinal traction. (Bottom left) Corresponding infrared image with the green line representing the level of the optical coherence tomography (OCT) scan. (Bottom right). The horizontal OCT section shows a LMH with intraretinal schisis and cavitation.

vascular arcades. Restaining to ascertain the complete removal of the ILM in the intended area was performed in all cases.

Finally, after careful inspection of the periphery over 360 degrees, fluid-air exchange was conducted in all cases to reduce the risk of postoperative hypotony. ${ }^{18,19}$

- STATISTICAL ANALYSIS: We compared the characteristics of eyes with and without LHEP using a $t$ test for continuous variables and a $\chi^{2}$ test for dichotomous variables. The correlation between BCVA and morphometric continuous variables was estimated after excluding the postoperative phase, using standardized coefficients in Structural Equation Models that account for repeated measures within individuals. Linear mixed models, with individuals as a random effect, were used to estimate the preoperative and postoperative changes in functional and anatomic variables, as well as the effects of covariates of interest. A $P$ value of less than .05 was considered significant. All analyses were conducted using the Stata 14.1 software (StataCorp 2015, College Station, Texas, USA).

\section{RESULTS}

A TOTAL OF 84 EYES FROM 84 PATIENTS MET THE INCLU. sion criteria and our definition of lamellar macular hole and were enrolled in the study. The mean age $( \pm$ standard deviation) was $67 \pm 7$ years. Of the 84 included patients, $35(41.7 \%)$ were male and $49(58.3 \%)$ were female. At baseline, 68 eyes were phakic and 16 eyes were pseudophakic; during the follow-up, 16 phakic eyes underwent cataract extraction and IOL implant without complications.

An analysis of OCT images at baseline showed LHEP in 41 eyes (48.8\%) and ERM in 73 eyes (86.9\%). Thirty eyes (35.7\%) presented with both LHEP and ERM (Figure 4); this association was statistically significant $(P<.001)$. Standard ERM alone was found in $43(51.2 \%)$ eyes, whereas LHEP alone was found in $11(13.1 \%)$ eyes. No eyes in this series did not present either LHEP or ERM.

At baseline, the group with LHEP $(n=41)$ and the group without LHEP $(n=43)$ differed with respect to the following variables: $\operatorname{logMAR}$ BCVA $(0.35 \pm 0.28$ vs $0.16 \pm 0.15, P<.001)$, CFT $(163 \pm 48$ vs $196 \pm 36, P$ $<.001)$, evidence of retinal wrinkling (37\% vs $67 \%, P=$ .002 ), and disruption of the outer retinal bands, which was noted in only 1 eye with ERM alone and in 27 eyes with LHEP $(65.8 \%, P<.001)$.

The Table summarizes the OCT and B-FAF findings of the sample at baseline.

Approximately one third of the patients underwent vitrectomy $(26 / 84,31 \%)$. Fourteen of the 26 operated eyes presented with standard ERM alone, 8 with LHEP and standard ERM, and 4 with LHEP alone. Among the patients who underwent surgery, 16 were operated immediately after the first examination, 5 were operated within 6 months, and 5 were operated between 6 and 24 months after the first visit.

Overall, the mean follow-up of patients who did not undergo surgery was $33.4 \pm 19.3$ months, whereas the postoperative follow-up of the operated patients was $32.8 \pm$ 21.6 months. The mean number of visits was $3.9 \pm 1.3$ in the nonoperated group and $4.3 \pm 1.3$ after surgery in the operated group.

- CORRELATIONS BETWEEN FUNCTIONAL AND ANATOMIC VARIABLES: We explored the cross-sectional correlations between functional and morphometric continuous variables after excluding the postoperative follow-up period in operated eyes, using all observational data and accounting for repeated measures within individuals. There was a strong correlation between B-FAF and OCT regarding both the horizontal and vertical diameters of 

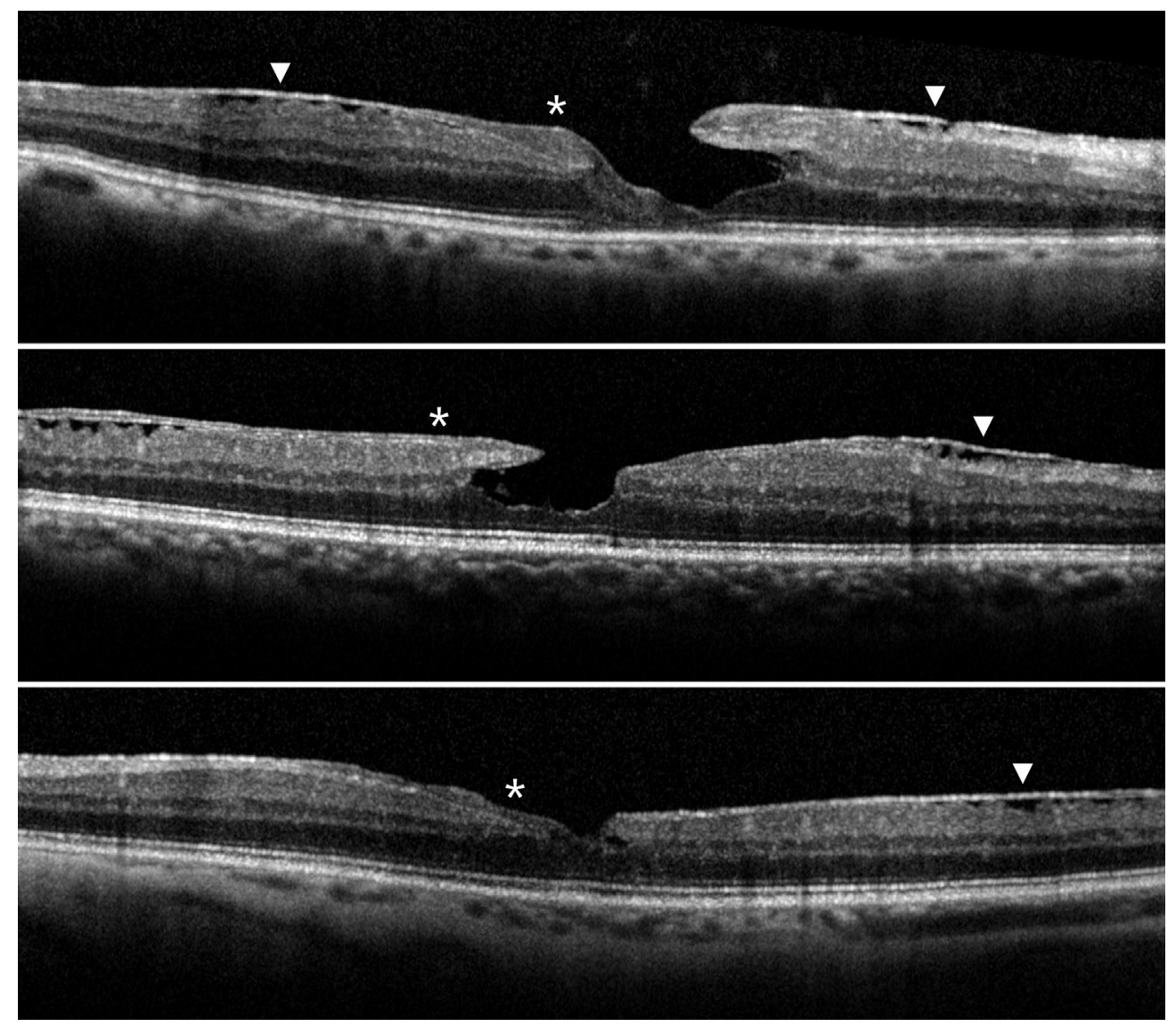

FIGURE 4. Concomitant presence of standard epiretinal membranes (ERM) and lamellar hole-associated epiretinal proliferation (LHEP) in eyes with lamellar macular holes. (Top, Middle, and Bottom) Standard ERMs appear as thin and highly reflective lines (arrowheads), whereas LHEPs (asterisks) appear as a homogenous material of medium reflectivity with focal variations in thickness and that conform to the adjacent retinal anatomy.

the holes ( $r=0.74$ and $r=0.76$, respectively, $P<.001$ ). The logMAR BCVA did not correlate with LMH diameters (Pearson $r$ between 0.01 and 0.18 ) but correlated moderately with CFT $(r=-0.32, P<.001)$.

In regression analyses with the baseline logMAR BCVA as the response variable, there was no significant interaction between LHEP and ERM in their association with visual acuity. However, in a model with no interaction term, LHEP accounted for a $0.19 \log$ MAR worse BCVA $(P=$ .001 ), while ERM was not associated with the BCVA (0.0 logMAR difference, $P=.955)$. Similarly, there was no interaction between LHEP and ERM in their association with CFT, but in a model with no interaction term, LHEP was associated with $-27 \mu \mathrm{m}$ CFT $(P=.007)$, while ERM accounted for $+21 \mu \mathrm{m}$ CFT $(P=.159)$. A disruption of the ELM, EZ, or IZ was associated with a 0.24-0.27 $\operatorname{logMAR}$ worse BCVA and a -50 to $-0.54 \mu \mathrm{m}$ foveal thickness $(P<.001$ in all cases $)$.

Neither LHEP nor ERM, nor their coexistence, was associated with hole diameters, as measured with B-FAF or OCT.
- FUNCTIONAL AND ANATOMIC EVOLUTION IN NONOP. ERATED AND OPERATED EYES BEFORE SURGERY: Figures 5 and 6 show the changes that occurred over time in the groups with and without LHEP and in the groups that underwent or did not undergo surgery relative to the following variables: $\log$ MAR BCVA, CFT, horizontal and vertical hole diameters measured with B-FAF and OCT imaging. The data were calculated up to 48 months, when at least 10 patients were followed up overall, making the mean values estimable.

A substantial stability of all parameters throughout the follow-up period was observed in the eyes that did not undergo surgery (Figures 7 and 8). A qualitative analysis of the images showed an enlargement/deepening of the holes in 10 of 41 eyes with LHEP and in 16 of 43 eyes with ERM alone, while spontaneous improvement was observed in 2 and 4 eyes, respectively. Three LMHs in the LHEP group evolved to full-thickness macular holes.

Baseline logMAR BCVA and the presence of LHEP were not significant predictors of changes in BCVA and CFT during the follow-up period. 
TABLE. Best-corrected Visual Acuity, Blue-Fundus Autofluorescence/Optical Coherence Tomography Findings, and Type of Epiretinal Materials Associated With Lamellar Macular Holes at Baseline

\begin{tabular}{|c|c|c|c|c|}
\hline & Total $(\mathrm{N}=84)$ & $\operatorname{LHEP}(\mathrm{N}=41)$ & No LHEP $(\mathrm{N}=43)$ & \\
\hline & Mean (SD) & Mean (SD) & Mean (SD) & $P$ Value \\
\hline BCVA (logMAR) & $0.25(0.25)$ & $0.35(0.28)$ & $0.16(0.15)$ & $<.001^{* a}$ \\
\hline B-FAF area $\left(\mathrm{mm}^{2}\right)$ & $0.22(0.15)$ & $0.23(0.18)$ & $0.20(0.16)$ & $.364^{a}$ \\
\hline B-FAF horizontal diameter $(\mu \mathrm{m})$ & $462(192)$ & $478(210)$ & $445(174)$ & $.434^{a}$ \\
\hline B-FAF vertical diameter $(\mu \mathrm{m})$ & $454(162)$ & $478(177)$ & $429(145)$ & $.166^{a}$ \\
\hline OCT horizontal diameter $(\mu \mathrm{m})$ & $445(167)$ & $476(174)$ & $415(156)$ & $.095^{a}$ \\
\hline OCT vertical diameter $(\mu \mathrm{m})$ & $399(159)$ & $428(169)$ & $371(146)$ & $.095^{a}$ \\
\hline OCT central foveal thickness $(\mu \mathrm{m})$ & $180(45)$ & $163(48)$ & $196(36)$ & $<.001^{* a}$ \\
\hline Posterior cortex visible on OCT & $20(24 \%)$ & $8(20 \%)$ & $12(28 \%)$ & $.367^{b}$ \\
\hline Standard ERM & $73(87 \%)$ & $30(36 \%)$ & $43(100 \%)$ & $<.001^{\star b}$ \\
\hline Retinal vessel printings & $10(12 \%)$ & $3(7 \%)$ & $7(16 \%)$ & $.205^{b}$ \\
\hline Retinal wrinkling & $44(52 \%)$ & $15(37 \%)$ & $29(67 \%)$ & $.002^{\star b}$ \\
\hline ORB disruption on OCT & $28(33 \%)$ & 27 (66\%) & $1(2 \%)$ & $<.001^{* b}$ \\
\hline \multicolumn{5}{|c|}{$\begin{array}{l}\mathrm{BCVA}=\text { best-corrected visual acuity; } \mathrm{B}-\mathrm{FAF}=\text { blue fundus autofluorescence; } \mathrm{ERM}=\text { epiretinal membrane; } \mathrm{LHEP}=\text { lamellar hole-associated } \\
\text { epiretinal proliferation; logMAR = logarithm of the minimum angle of resolution; OCT = optical coherence tomography; ORB = outer retinal } \\
\text { bands. } \\
P \text { values with asterisk are statistically significant. } \\
{ }^{a} t \text { test } P \text { value. } \\
{ }^{b} \chi^{2} P \text { value. }\end{array}$} \\
\hline
\end{tabular}

In the group that underwent surgery after a follow-up period between 3 and 24 months $(n=10)$, a significant change in the following variables, which were evaluated during the preoperative period, was observed: BCVA (0.077 logMAR per 12 months, $P=.015)$; FAF vertical diameter $(+70 \mu \mathrm{m}$ per 12 months, $P=.004)$; and CFT $(-51 \mu \mathrm{m}$ per 12 months, $P<.001)$.

- POSTOPERATIVE COURSE IN OPERATED EYES: Twentysix eyes underwent surgery (Figures 9 and 10). The logMAR BCVA improved continuously during the postoperative period, with a linear trend of $0.035 \log \mathrm{MAR}$ per 12 months $(P=.006)$. The central foveal thickness was found to increase, and the horizontal and vertical diameters of the holes, measured with B-FAF and OCT imaging, were found to be reduced starting from the first month after the operation $(P<.001)$, with no detectable trend of change afterwards. These anatomic improvements were observed both in the eyes with standard ERM (associated or not with LHEP) and in the 4 eyes with LHEP alone.

Closure of the hole was achieved in the 3 cases of LMH that evolved to full-thickness macular holes and underwent surgery. One of these eyes presented with LHEP alone (Figure 11).

- PREDICTORS OF FUNCTIONAL OUTCOMES IN OPERATED EYES: The effect of preoperative variables on the change of the logMAR BCVA in the 26 operated eyes was investigated. Only univariate associations were explored owing to the small number of patients, adjusting only for the effect of linear time. As expected, the preoperative BCVA was a strong predictor of postoperative visual change $(P=.001)$. When the linear effect of the baseline BCVA was explored, the model predicted no improvement at a baseline visual acuity of $0.24 \log$ MAR and an improvement of 0.1 for each $0.2 \log$ MAR worse BCVA. We did not investigate nonlinear effects owing to the small sample size. No other clinical finding was a significant predictor of BCVA change after surgery, including the presence of ERM or LHEP. Specifically, LHEP did not influence the linear trend of the postoperative BCVA.

\section{DISCUSSION}

IN THIS RETROSPECTIVE STUDY, WE SHOW THAT IN EYES with LMHs, standard ERMs are found much more frequently than LHEP ( $86.9 \%$ vs $48.8 \%$ ), and the presence of LHEP without any detectable standard ERM is rare (13.1\% of cases). In approximately one third of cases, a standard ERM and LHEP coexist.

Lamellar macular holes with LHEP are characterized by a poorer BCVA, a thinner CFT, and disrupted outer retinal bands in comparison to LMHs with standard ERM alone, but the horizontal and vertical diameters of the holes, as measured with B-FAF or with OCT at the level of the outer plexiform layer, do not differ in the 2 groups. Independently from the presence of LHEP, most of the eyes with LMHs tend to remain stable during years-long follow-up, 

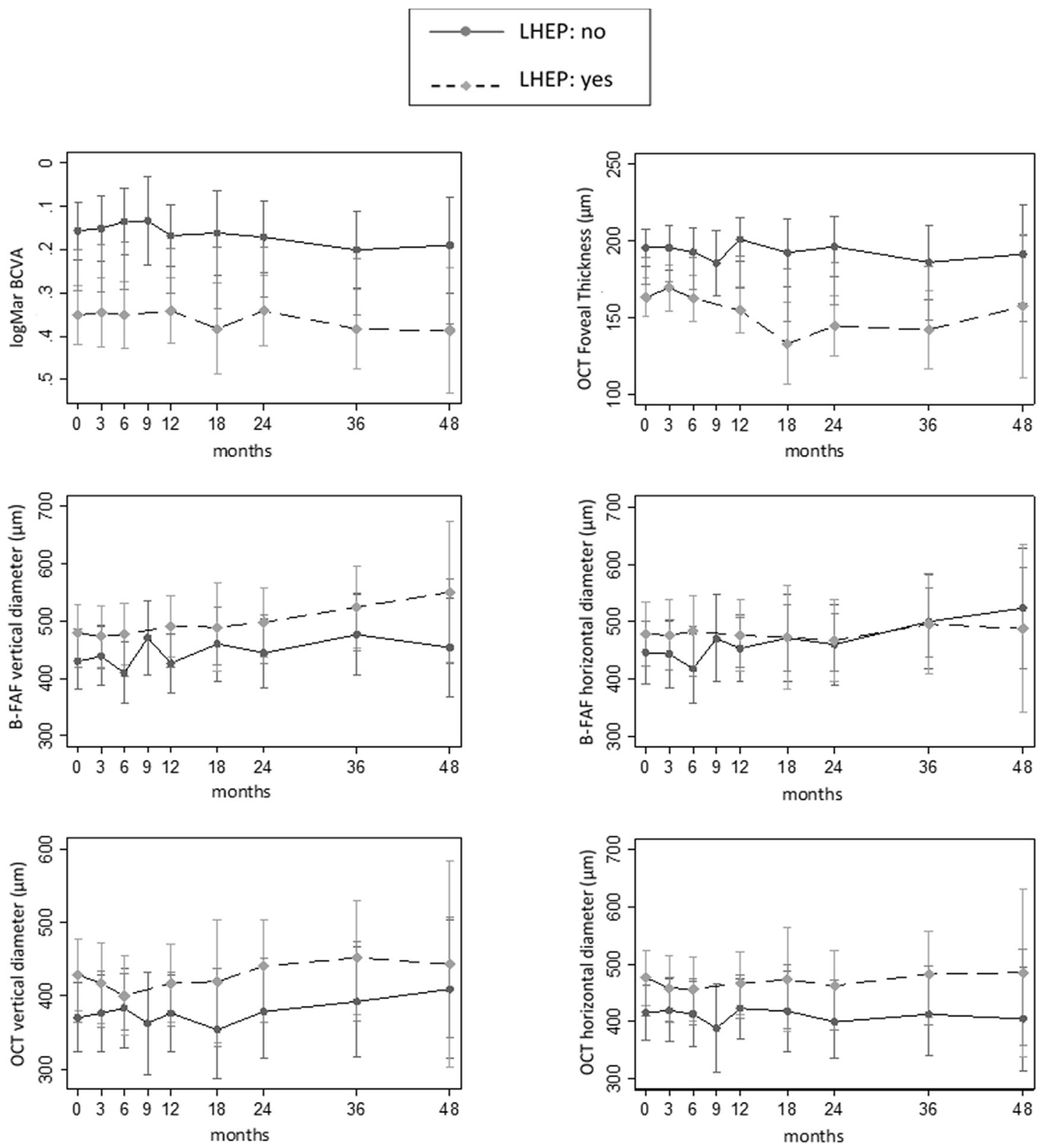

FIGURE 5. Baseline and follow-up values (vertical bar: standard error) of best-corrected visual acuity (BCVA), central foveal thickness, and hole diameters measured with blue-fundus autofluorescence (B-FAF) and optical coherence tomography (OCT) in eyes with ( $n=41$, dashed line) and without lamellar hole epiretinal proliferation (LHEP, $n=43$, solid line). Estimates are derived from a linear mixed model.

and morphologic and functional outcomes after surgery do not differ in cases with and without LHEP.

Recently, the enhanced resolution of SDOCT has enabled the recognition of 2 types of epiretinal material that can be detected in association with LMHs: (1) standard ERM, which is a hyperreflective line that is usually accompanied by wrinkling of the underlying retinal surface, and (2) LHEP, which is a thick homogenous layer of material with medium reflectivity that conforms to the retinal anatomy. ${ }^{1,5,7-10}$
The concomitant presence of a standard ERM and LHEP in association with LMHs has been reported variably in the literature, with values ranging from $10.78 \%$ to $46 \%$.

We found this association in $35.7 \%$ of the examined eyes. In line with previous observations, standard ERMs were more common than LHEP, with only 11 of 84 eyes showing no trace of standard ERM. ${ }^{1,6,7}$

It must be noted that identifying the presence of trace amounts of LHEP in cases with a prominent, standard ERM and vice versa may be difficult and easily 

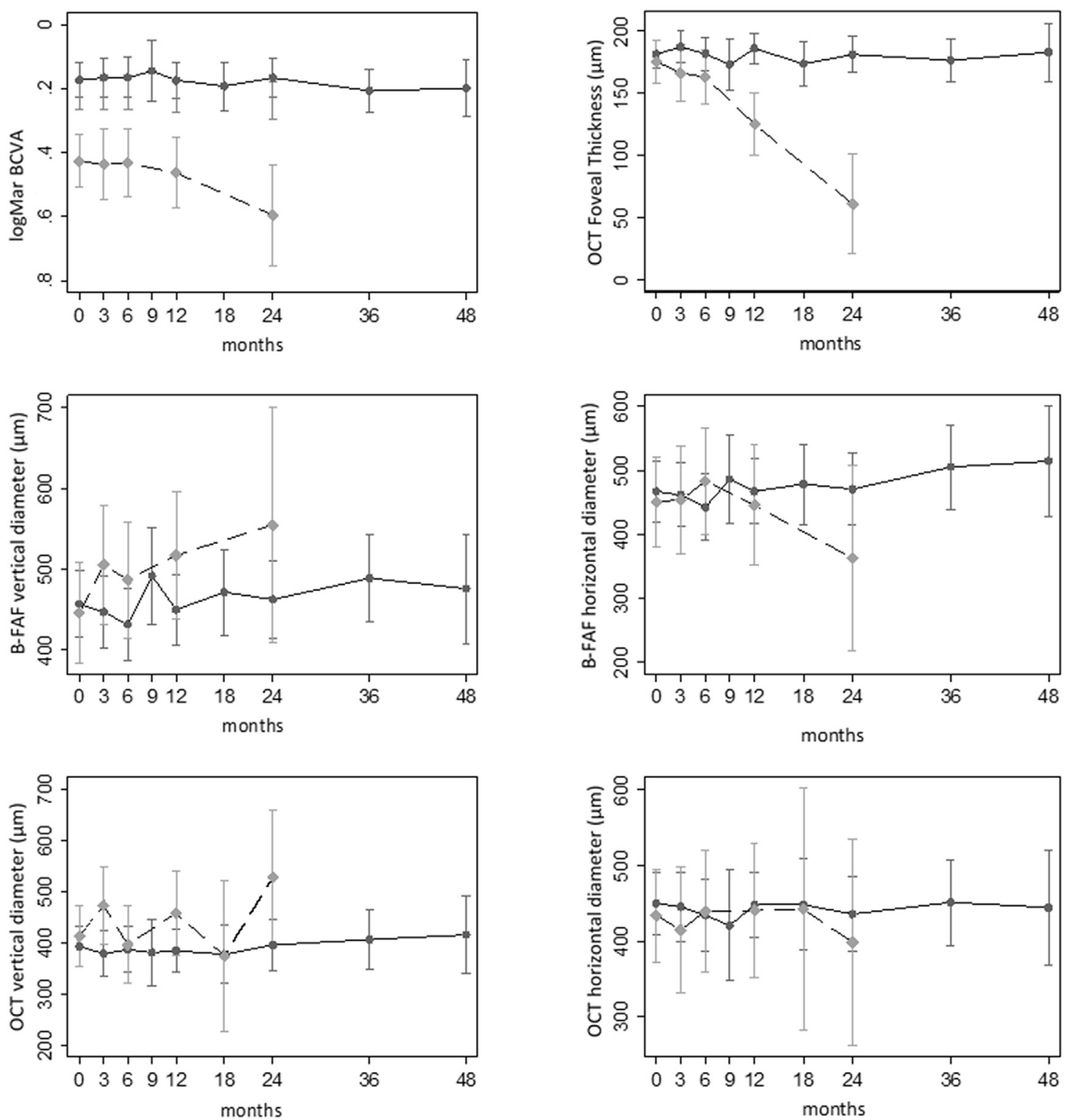

FIGURE 6. Baseline and follow-up values (vertical bar: standard error) of best-corrected visual acuity (BCVA), central foveal thickness, and hole diameters measured with blue-fundus autofluorescence (B-FAF) and optical coherence tomography (OCT) in nonoperated $(n=58$, solid line) and operated eyes $(n=26$, dashed line). Estimates are derived from a linear mixed model.

underestimated if the OCT scans do not encompass the entire macular area or if few OCT scans are made. Therefore, the density, orientation, and number of B-scans recorded are crucial factors for confirming or excluding the presence of each type of epiretinal material in association with an LMH. For this reason, strict categorization of LMHs based solely on the associated epiretinal material should be avoided because in several cases, 1 type of epiretinal material is predominant but not exclusive. ${ }^{7,9}$

The presence of LHEP does influence some morphologic and functional features of LMHs.

For example, in line with previous studies, ${ }^{5-7,9,10,12}$ we found that a worse BCVA, a thinner CFT, and the disruption of the outer retinal bands were observed more 


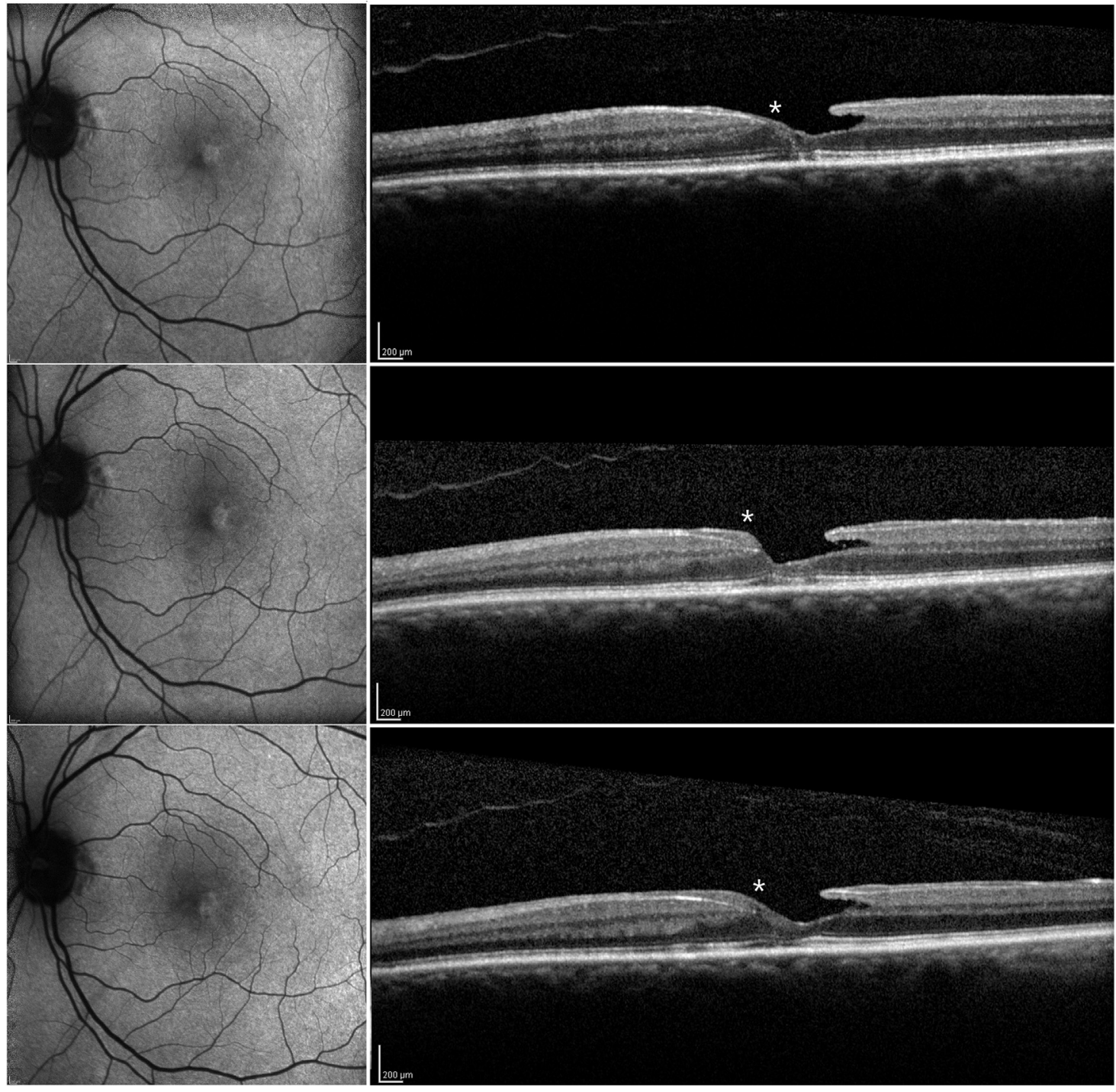

FIGURE 7. Serial blue-fundus autofluorescence (B-FAF) and eye-tracked optical coherence tomography (OCT) images of a 63-yearold woman affected by lamellar macular hole with lamellar hole-associated epiretinal proliferation (LHEP) showing progression of the LHEP lesion (asterisks) and stability of the B-FAF features over the duration of 48 months of retrospective follow-up. (Top left) B-FAF and (Top right) OCT images at baseline. (Middle left) At 24 months of follow-up there is no change in the appearance of B-FAF, whereas the LHEP appears thickened on OCT (Middle right). (Bottom left) At 48 months from baseline, features of B-FAF appear stable, whereas the LHEP observed on OCT has further thickened (Bottom right).

often in the presence of LHEP. Furthermore, a cavitated appearance of the retina was frequently found in association with LHEP, while standard ERMs were usually associated with a schitic lamellar separation of the retina.

On the other hand, other aspects appeared to be similar in LMHs with and without LHEP. For example, as reported by Schumann and associates, ${ }^{7}$ a vitreal posterior cortex, detached from the macular area and visible on OCT scans, was observed in a similar percentage of eyes with and without LHEP. Measurements of the horizontal and vertical diameters of the holes at the level of the outer plexiform layer recorded on OCT or the horizontal and vertical diameters of the holes measured on B-FAF images did not differ between eyes with and without LHEP at baseline and did not change significantly during the follow-up period. Similarly, the mean CFT did not change significantly during the follow-up period in either group, as reported by Govetto and associates.

The fact that LMHs with and without LHEP may share some similarities also emerges from histopathology studies. $^{10,11}$ These studies have demonstrated that the constituents of standard ERM and LHEP are the same, although the predominance of some of those constituents may vary according to the type of epiretinal material. Specifically, it has been shown that glial fibrillary acidic 


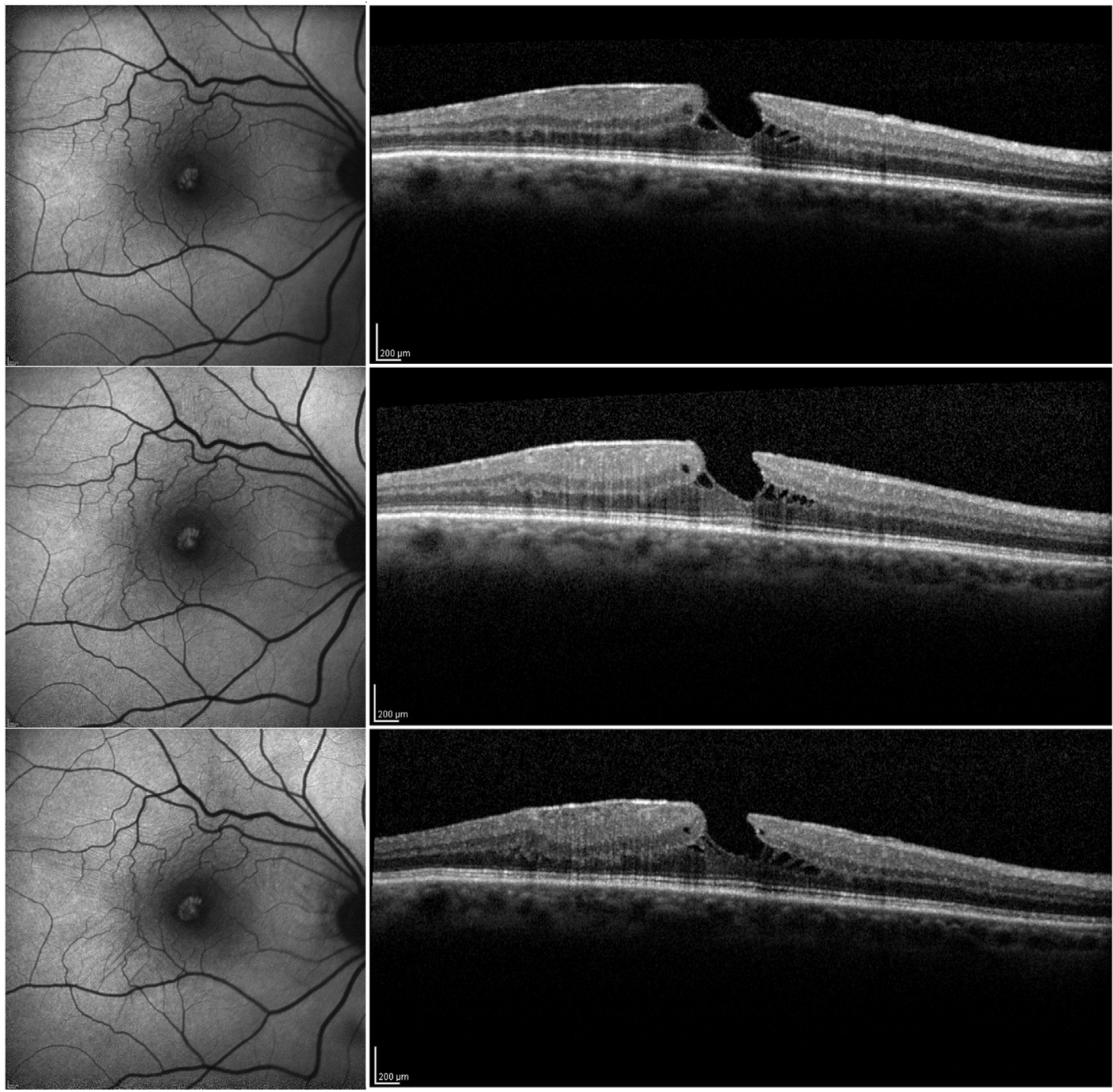

FIGURE 8. Serial blue-fundus autofluorescence (B-FAF) and eye-tracked optical coherence tomography (OCT) images of a 71-year-old man affected by lamellar macular hole and standard epiretinal membrane, showing substantial stability of both B-FAF and OCT features over the duration of 48 months of retrospective follow-up. (Top, left and right). B-FAF and OCT images taken at baseline. (Middle, left and right). B-FAF and OCT images taken at 24 months of follow-up. (Bottom, left and right). B-FAF and OCT images taken at 48 months of follow-up.

protein is commonly detected both in standard ERM and LHEP, but fibrous long-spacing collagen and collagen type I and III predominate in LHEP, whereas $\alpha$-smooth muscle actin $(\alpha-S M A)$ is expressed more frequently in standard ERMs.

Because the presence of $\alpha$-SMA would confer contractile properties to the epiretinal material, we investigated the presence of RVPs, which are a useful sign for indirectly evaluating the tangential traction related to epiretinal membranes on B-FAF imaging. ${ }^{16,17}$

Our results appear to confirm the scarce contractile characteristics of LHEP because RVPs were detected in none of the 11 eyes presenting with LHEP alone. Conversely, RVPs were found in $16.3 \%$ of the eyes with standard ERM alone and in an additional $7.3 \%$ of eyes with the coexistence of LHEP and ERM.

While in the nonoperated eyes morphometric and functional parameters remained generally stable over the course of follow-up, in the group that eventually underwent surgery the BCVA and CFT decreased and the vertical diameter of the hole measured with B-FAF increased significantly from the baseline examination to the visit performed before the operation. The most significant change, observed both in eyes with and in those without LHEP, was represented by the progressive thinning of the CFT. Thus, careful examination of the OCT scans passing 


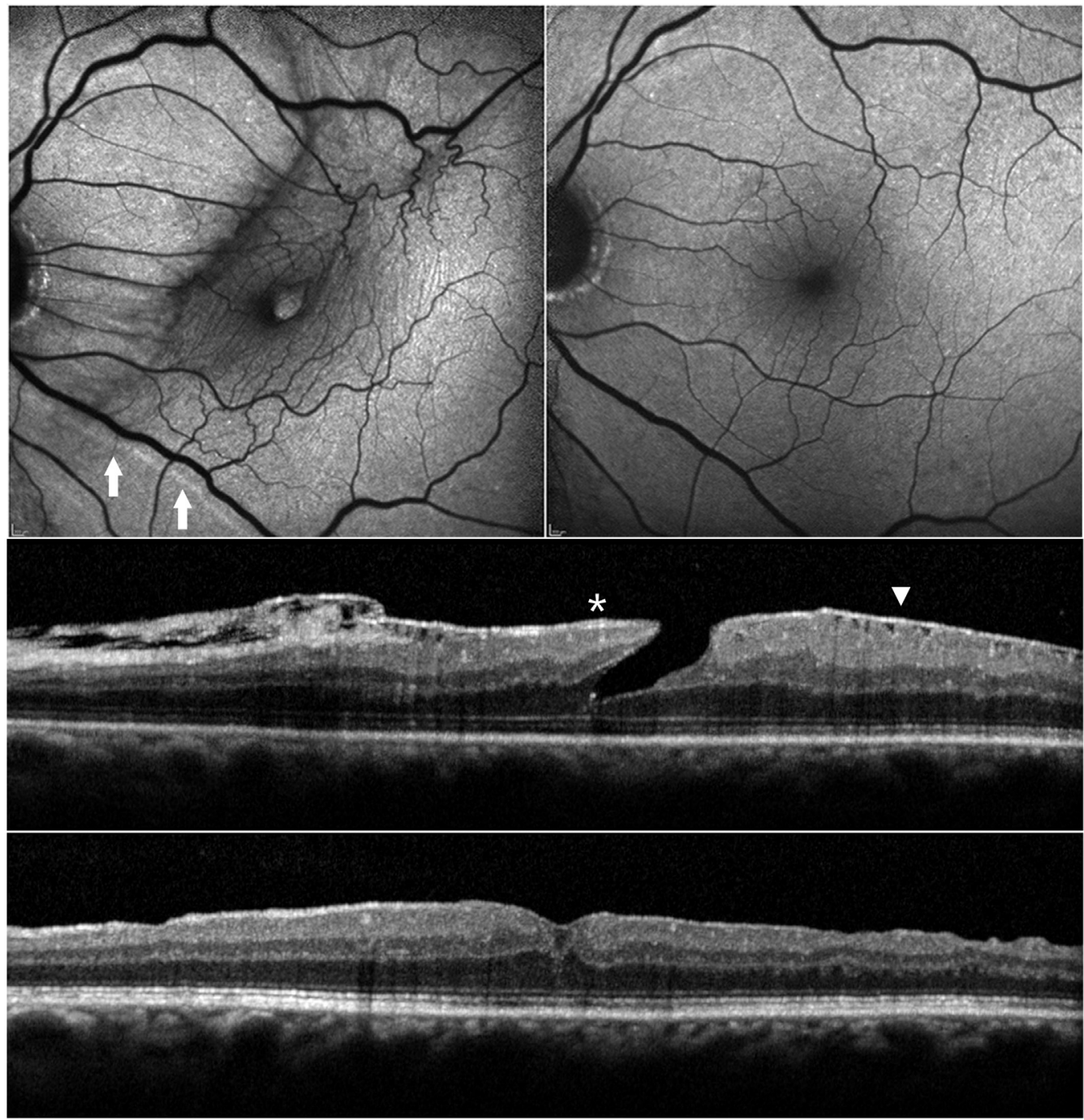

FIGURE 9. Evolution of blue-fundus autofluorescence (B-FAF) and optical coherence tomography (OCT) features after vitrectomy with peeling of epiretinal membranes and inner limiting membrane. (Top left) Preoperative B-FAF shows distorted retinal vessels, retinal vessel printings (RVPs, arrows), and hyperautofluorescence at the level of the hole. (Top right) Three months after operation the retinal vasculature has recovered its normal appearance, the RVPs are no longer visible, and the central area of hyperautofluorescence has disappeared. (Middle) Preoperative OCT shows cavitation of the retina at the fovea, presence of lamellar hole-associated epiretinal proliferation (asterisk), and standard epiretinal membrane (arrowhead). (Bottom). Three months after operation the OCT shows a relatively smooth retinal surface and a partially restored foveal contour.

through the foveal center and repeated measurements comparing the thickness of the central foveal tissue at the same location over time are recommended in eyes with LMHs when looking for signs of progression.

After surgery, thickening of the central fovea, reduction of the horizontal and vertical diameters of the holes on OCT, and reduction of the horizontal and vertical diameters of the area of increased autofluorescence on B-FAF were observed after the first postoperative month in all eyes, independent of the type of associated epiretinal material.

Interestingly, we noted such evolution also in the 4 eyes with LHEP alone that underwent surgery. This result may suggest that LHEP, a tissue supposed to have minimal contractile properties, may actually assert some kind of traction onto retinal layers, causing distortion of the foveal architecture and damage to retinal cells. Surgical removal of LHEP, relieving this traction, could promote the restoration of a normal foveal anatomy and function. Alternatively, it is possible that the contraction of the ILM is primarily responsible for the distorted foveal contour and reduced visual acuity. In eyes with LMH and LHEP, the contraction of the ILM could be masked and easily underestimated preoperatively because LHEP has a smooth inner surface and is a thick material that perfectly moulds to the superficial retinal irregularities and 


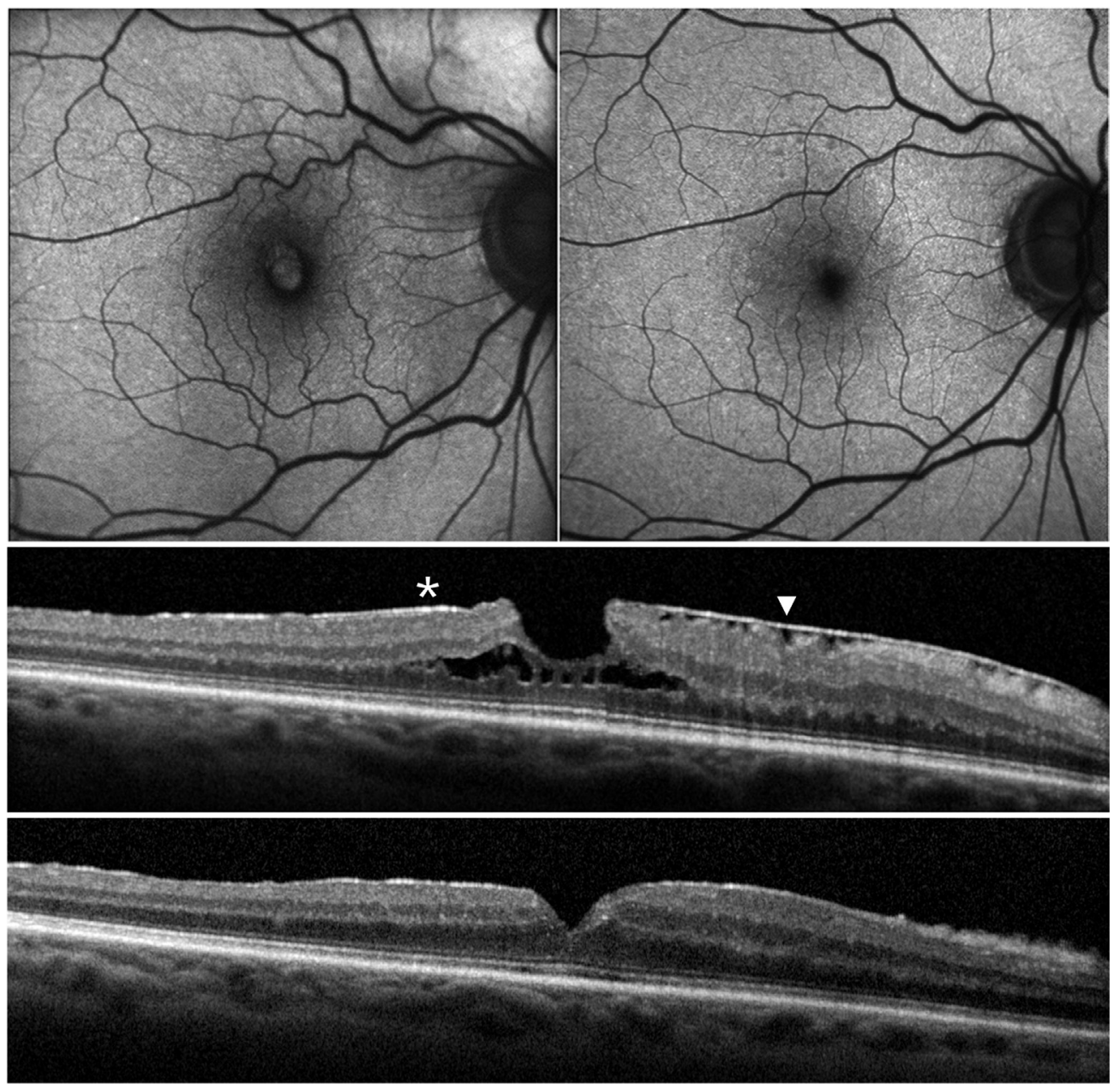

FIGURE 10. Evolution of blue-fundus autofluorescence (B-FAF) and optical coherence tomography (OCT) features after vitrectomy with peeling of epiretinal membranes and inner limiting membrane. (Top left) Preoperative B-FAF shows distorted retinal vessels and hyperautofluorescence at the level of the hole. (Top right) Three months after operation the retinal vasculature has recovered its normal appearance and the area of hyperautofluorescence has disappeared. (Middle) Preoperative OCT shows the presence of lamellar hole-associated epiretinal proliferation (asterisk) and standard epiretinal membrane along with the schitic lamellar separation of the retina. (Bottom) Three months after operation the OCT shows a relatively smooth retinal surface and a partially restored foveal contour.

undulations, making them undetectable. Thus, peeling of LHEP might be not as important for relieving epiretinal traction as peeling a standard ERM, but it would have the function of allowing surgical access to the ILM. The role of ILM as epiretinal material exerting traction in association with LMHs seems to be also confirmed by the fact that, as reported by previous studies, ${ }^{5,12}$ and as observed in this series, full-thickness macular holes evolved from LMHs and associated with LHEP generally close after surgical removal of ILM.

The origin of the area of increased autofluorescence observed in association with LMHs on B-FAF imaging is not clear. This feature might represent an actual loss of foveal tissue or a mere centrifugal displacement of neurosensory tissue containing macular pigment. ${ }^{4,15,20}$ At present, it appears that neither OCT nor B-FAF can answer this question.
Whatever its origin, the increased autofluorescent signal at the fovea in eyes with LMHs appeared to be similar in size in the presence of standard ERM or LHEP and was reduced in eyes with and without LHEP after surgery. Thus, LMHs with different features based on OCT may appear to be indistinguishable based on FAF imaging.

Visual acuity improvements after surgery were recorded not only in eyes with standard ERM but also in eyes with LHEP. Thus, despite a lower preoperative BCVA, a thinner CFT, and disrupted outer retinal bands, eyes with LHEP might benefit from surgery as much as eyes with standard ERM. This finding is important in light of the fact that recommendations for surgical repair of eyes with LMHs are controversial, with some authors reporting good surgical outcomes and others documenting worsening of anatomic features and VA after surgery. ${ }^{1,3,7,10,11,21-23}$ 


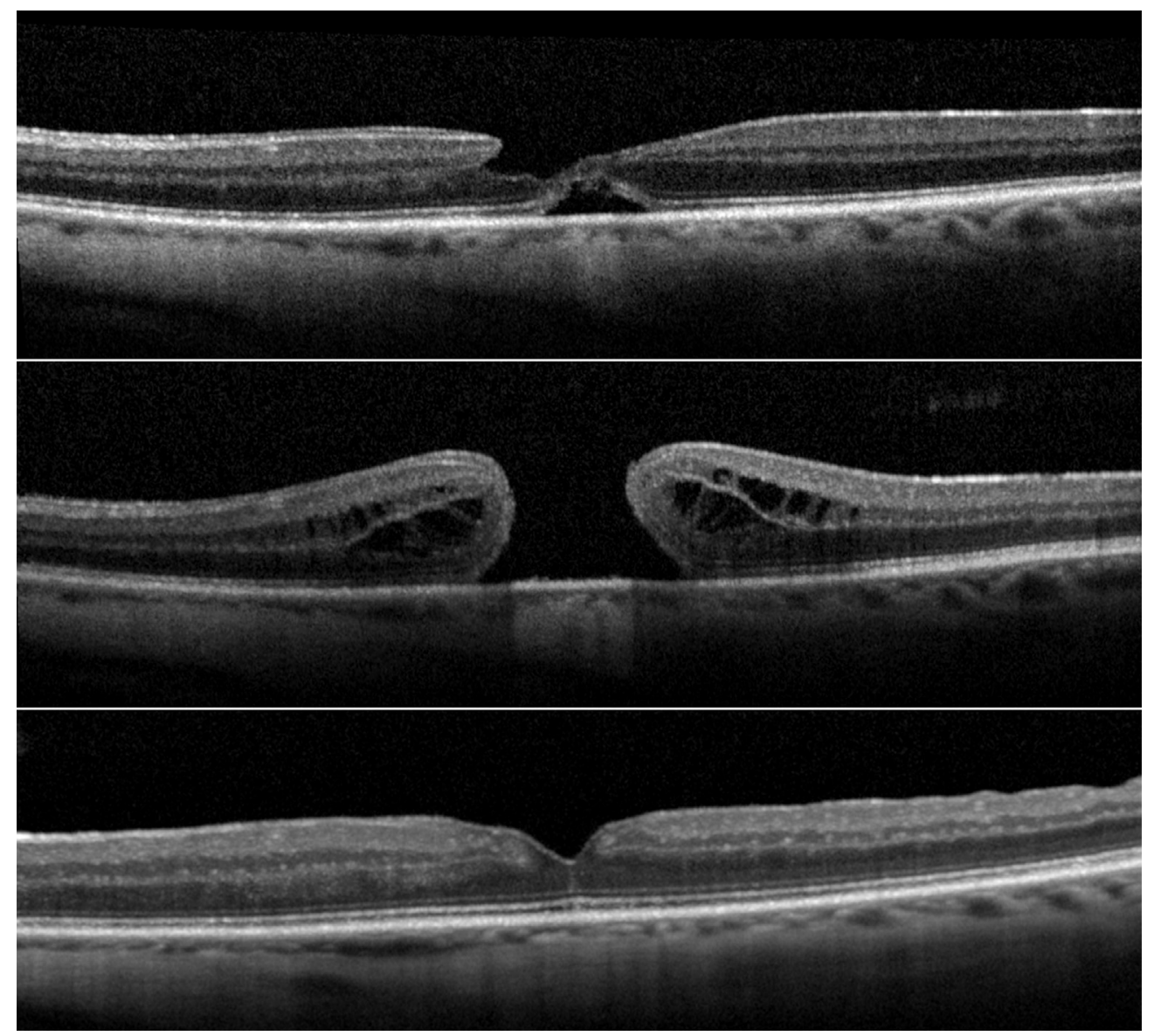

FIGURE 11. Serial eye-tracked optical coherence tomography (OCT) images showing evolution of lamellar macular hole (LMH) to full-thickness macular hole (FTMH) and outcome after vitrectomy and peeling of epiretinal membranes and inner limiting membrane. (Top) LMH with lamellar hole-associated epiretinal proliferation. (Middle) Two months later the LMH has evolved to FTMH. (Bottom) Three months after operation, closure of the macular hole is observed.

The limitations of this study include its retrospective nature, the relatively restricted number of patients, and the variable duration of follow-up.

The strengths of this study include the use of an OCT device that is able to record serial images at the same location, the inclusion of several follow-up visits for each patient, and the simultaneous use of B-FAF and OCT.
To conclude, we show that in eyes with LMHs, the presence of LHEP without any trace of standard ERM is rare and that standard ERMs and LHEPs coexist in at least one third of cases. The type of epiretinal proliferation may be associated with specific features on OCT but does not appear to influence the size of the area of increased autofluorescence on B-FAF imaging, the natural course of the disease, or the response to surgery.

FUNDING/SUPPORT: NO FUNDING OR GRANT SUPPORT. FINANCIAL DISCLOSURES: THE FOLLOWING AUTHORS HAVE NO financial disclosures: Roberto dell'Omo, Gianni Virgili, Stanislao Rizzo, Serena De Turris, Giovanni Coclite, Dario Giorgio, Ermanno dell'Omo, and Ciro Costagliola. All authors attest that they meet the current ICMJE criteria for authorship.

\section{REFERENCES}

1. Witkin AJ, Ko TH, Fujimoto JG, et al. Redefining lamellar holes and the vitreomacular interface: an ultrahigh resolution optical coherence tomography study. Ophthalmology 2006; 113(3):388-397.
2. Michalewska Z, Michalewski J, Odrobina D, Nawrocki J. Nonfull-thickness macular holes reassessed with spectral domain optical coherence tomography. Retina 2012;32(5):922-929.

3. Gaudric A, Aloulou Y, Tadayoni R, Massin P. Macular pseudoholes with lamellar cleavage of their edge remain pseudoholes. Am J Ophthalmol 2013;155(4):733-742. 
4. Staurenghi G, Sadda S, Chakravarthy U, Spaide RF, for the International Nomenclature for Optical Coherence Tomography (IN OCT) Panel. Proposed lexicon for anatomic landmarks in normal posterior segment spectral-domain optical coherence tomography: the IN.OCT consensus. Ophthalmology 2014;121(8):1572-1578.

5. Pang CE, Spaide RF, Freund KB. Epiretinal proliferation seen in association with lamellar macular holes: a distinct clinical entity. Retina 2014;34(8):1513-1523.

6. Pang CE, Spaide RF, Freund BK. Comparing functional and morphologic characteristics of lamellar macular holes with and without lamellar hole-associated epiretinal proliferation. Retina 2015;35(4):720-726.

7. Schumann RG, Compera D, Schaumberger MM, et al. Epiretinal membrane characteristics correlate with photoreceptor layer defects in lamellar macular holes and macular pseudoholes. Retina 2015;35(4):727-735.

8. Itoh Y, Levison AL, Kaiser PK. Prevalence and characteristics of hyporeflective preretinal tissue in vitreomacular interface disorders. Br J Ophthalmol 2016;100(3):399-404.

9. Govetto A, Dacquay Y, Farajzadeh M, et al. Lamellar macular hole: two distinct clinical entities? Am J Ophthalmol 2016; 164:99-109.

10. Parolini B, Schumann RG, Cereda MG, Haritoglou C, Pertile G. Lamellar macular hole: a clinicopathologic correlation of surgically excised epiretinal membranes. Invest Ophthalmol Vis Sci 2011;52(12):9074-9083.

11. Compera D, Entchev E, Haritoglou C, et al. Lamellar holeassociated epiretinal proliferation in comparison to epiretinal membranes of macular pseudoholes. Am J Ophthalmol 2015; 160(2):373-384.

12. Bottoni F, Deiro AP, Giani A, Orini C, Cigada M, Staurenghi G. The natural history of lamellar macular holes: a spectral domain optical coherence tomography study. Graefes Arch Clin Exp Ophthalmol 2013;251(2):467-475.

13. Delori FC, Dorey CK, Staurenghi G, Arend O, Goger DG, Weiter JJ. In vivo fluorescence of the ocular fundus exhibits retinal pigment epithelium lipofuscin characteristics. Invest Ophthalmol Vis Sci 1995;36(3):718-729.

14. von Rückmann A, Fitzke FW, Bird AC. Distribution of pigment epithelium autofluorescence in retinal disease state recorded in vivo and its change over time. Graefes Arch Clin Exp Ophthalmol 1999;237(1):1-9.

15. Bottoni F, Carmassi L, Cigada M, Moschini S, Bergamini F. Diagnosis of macular pseudoholes and lamellar macular holes: is optical coherence tomography the "gold standard"? Br J Ophthalmol 2008;92(5):635-639.

16. Nitta E, Shiraga F, Shiragami C, Fukuda K, Yamashita A, Fujiwara A. Displacement of the retina and its recovery after vitrectomy in idiopathic epiretinal membrane. Am J Ophthalmol 2013;155(6):1014-1020.

17. dell'Omo R, Cifariello F, dell'Omo E, et al. Influence of retinal vessel printings on metamorphopsia and retinal architectural abnormalities in eyes with idiopathic macular epiretinal membrane. Invest Ophthalmol Vis Sci 2013;54(12): 7803-7811.

18. Shimada H, Nakashizuka H, Mori R, Mizutani Y, Hattori T. 25-gauge scleral tunnel transconjunctival vitrectomy. Am J Ophthalmol 2006;142(5):871-873.

19. Bamonte G, Mura M, Tan HS. Hypotony after 25-gauge vitrectomy. Am J Ophthalmol 2011;151(1):156-160.

20. Bottoni F, Zanzottera E, Carini E, Cereda M, Cigada M, Staurenghi G. Re-accumulation of macular pigment after successful macular hole surgery. Br J Ophthalmol 2016;100(5): 693-698.

21. Garrettson BR, Pollack JS, Ruby AJ, Drenser KA, Williams GA, Sarrafizadeh R. Vitrectomy for a symptomatic lamellar macular hole. Ophthalmology 2008;115(5):884-886.

22. Sun JP, Chen SN, Chuang CC, et al. Surgical treatment of lamellar macular hole secondary to epiretinal membrane. Graefes Arch Clin Exp Ophthalmol 2013;251(12):2681-2688.

23. Michalewska Z, Michalewski J, Odrobina D, et al. Surgical treatment of lamellar macular holes. Graefes Arch Clin Exp Ophthalmol 2010;248(10):1395-1400. 